Systematic Review

\title{
Analysis of Occupational Therapy In Schizophrenic Patients
}

\author{
Siti Khadijah, Darni Darni, and Sitti Sulaihah
}

Faculty of Nursing, Universitas Airlangga, Surabaya, Indonesia

\begin{abstract}
Introduction: Nursing care in the rehabilitation phase is one which provides occupational therapy, which is one form of combination therapy between art and science to direct patients to selective activities, so that health can be improved and maintained, and prevent disability through work activities and training This study is to show evidence of the effectiveness of occupational therapy interventions: job training in schizophrenic patients at the Mental Hospital.
\end{abstract}

Methods: The method used in this systematic review consists of 5 stages: identification of instruments in the literature (database search); identification of relevant literature based on title and abstract; inclusion and exclusion criteria; obtaining full text literature; assessment based on literature component and analysis of selected instruments. The database used in the literature search was Scopus, Proquest, Science Direct, Japan Journal, Cochrane by limiting the keywords "Occupational Therapy" and "Schizophrenia", which were published in the years between 2012-2018.

Results: Fifteen studies were included in the analysis, only 10 journals met the criteria to be included in the quantitative design.

Conclusion: From the results of this study it was found that occupational therapy was significant in improving cognitive, social, work, welfare, and contributing to the quality of life of patients.

\section{ARTICLE HISTORY}

Received: Dec 26, 2019

Accepted: Dec 31, 2019

\section{KEYWORDS}

occupational therapy; cognitive; social; work, welfare

\section{CONTACT}

Siti Khadijah

$\triangle$ khadije1704@gmail.com

$\fallingdotseq$ Faculty of Nursing, Universitas

Airlangga, Surabaya, Indonesia

Cite this as: Khadijah, S., Darni, D., \& Sulaihah, S. (2019). Analysis of Occupational Therapy In Schizophrenic Patients. Jurnal Ners, 14(3si), 336-339. doi:http://dx.doi.org/10.20473/jn.v14i3(si).17178

\section{INTRODUCTION}

Mental health is still one of the most significant health problems in the world, including in Indonesia. The World Health Organization (WHO) estimates that more than 21 million people worldwide are living with schizophrenia. The median incidence of schizophrenia is estimated at 15.2 cases per 100,000 of the general population with lifetime prevalence estimated at 7.2 per 1000 of the general population (McGrath 2008). Prevalence is higher in males compared to females (rate ratio 1.4:1) and the mortality risk for people with schizophrenia is two to three times that of the general population, with an allcause standardized mortality ratio of 2.6:1. The number of mental disorders is still very high. One of the most common mental disorders is schizophrenia which is a disease that affects the brain and results in disorders of the mind, perception (hallucinations), emotions, and behavior (Videbeck, Sheila, 2008)
Schizophrenia produces experiences of loss of work, since suffering from the disease affects quality of life and has a high rate of recurrence, (Singh, Singh, $\&$., 2019) explains the results of his research on studies showing that people who suffer from schizophrenia have poor maintenance abilities, their personal hygiene, loss of interest in meeting people at the time of illness, poor communication skills (good skill deficits). Scores also indicate that they lost their work skills since the disease began. Nursing care at the rehabilitation stage is one of which provides occupational therapy which is a combination of art and science to direct patients to selective activities, so that health can be improved and maintained, and prevent disability through activities and work activities for mentally and physically handicapped people (Badan Penelitian dan Pengembangan Kesehatan, 2013). This is supported by research journals from D'amico, (2018) which confirms that every impact of psychosocial therapy and rehabilitation, neutralizes the causes of withdrawal of 
patients from social life. Social skills training is needed in teaching patients to return to situations when they will be able to function properly in their environment (Amico, Jaffe, \& Gardner, 2018).

\section{MATERIALS AND METHODS}

\section{Literature Search Strategy}

The literature used in this Systematic Review uses tracing through 6 (six) electronic databases, namely: Scopus, Science Direct, Proquest, which has been published in publications from 2012 to 2018.

That is the key word used is Occupational Therapy and Schizophrenia. Furthermore, the method chosen was a study using randomized clinical trials (RCTs) which discussed occupational therapy in schizophrenia.

\section{Study design}

The study design became the inclusion criteria in this Systematic Review, the design is a Random Controlled Trial (RCT) published in English.

\section{Population}

The population in this systematic review is all studies evaluated patients who received occupational therapy interventions with or without a control group, such as schizophrenic patients, productive age according to WHO (15 - 54 years), and rehabilitation patients, able to communicate well and cooperatively.

\section{Intervention}

Occupational therapy that has been found in the study of this literature is given by a combination of social management, Psychosocial Interventions on Interpersonal Functioning and Personal and Social, Occupational Goal Intervention

\section{Clinical results}

The main outcome of the main objective of this systematic review is that occupational therapy interventions have a significant impact on improving cognitive, social, occupational and patient welfare functions, contributing to the quality of life of patients and effective treatment in schizophrenic patients.

\section{Study selection}

Standard protocols are used for selecting studies as suggested in the systematic review method guide, PRISMA. The steps taken are duplicate eraser, examination independently for titles, abstracts, and keywords and citations that are not relevant according to the inclusion criteria, $f$ the title and abstract are approved by the inclusion review meeting and are in accordance with the objectives of the systematic review, the next step is the selection of the journal with full text, the final step is to select a journal using a randomized controlled trial to reduce the possibility of bias

\section{Data extraction}

Data is extracted from each studio that meets the requirements. The extracted data adds research characteristics, characteristics of occupational

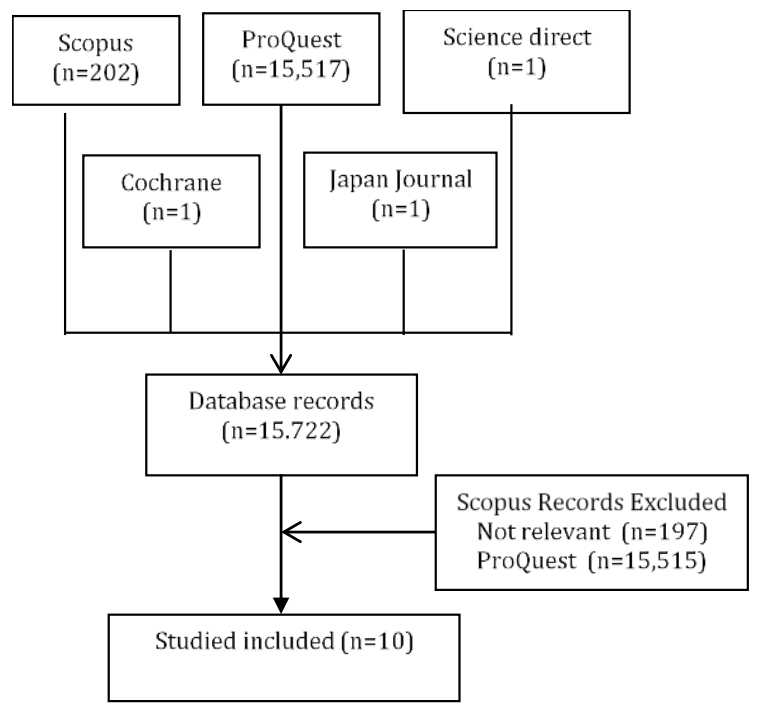

Figure 1. PRISMA of systematic review

therapy, characteristics of results and ranking of results.

\section{Data analysis}

Studies are grouped according to the effects of occupational therapy in improving cognitive, social, occupational and patient welfare functions, contributing to patients' quality of life and effective treatment in schizophrenic patients. If possible, the research was then grouped based on the time of follow-up and the type of control group.

\section{RESULTS}

Picture 1. The search results and study selection follow the PRISMA guidelines (Liberati et al., 2009). Selection of journals was based on the keywords used to produce Prequest 15,517 there are 2 corresponding journals and 15,515 journals issued because they do not meet the eligibility criteria, Scopus 202 there are 5 appropriate journals and 197 journals issued because they do not meet the eligibility criteria. Science direct has 1 journal that matches the criteria, Japan Journal of Nursing Science has 1 journal that is appropriate. In Cochrane there is 1 corresponding journal. A total of 10 studies were selected for systematic review.

The results of research show that social skills develop the potential to maintain socio-occupational skills personally with schizophrenia (Singh \& Singh, 2018). The results of Çakmak, et al, study that there are benefits from work activities, as indicated by improvements in all client psychiatry scores on Personal and Social Performance Scale (PSP) compared to scores from the control group: patients who did not participate in this activity (Çakmak, Süt, Öztürk, Tamam, \& Bal, 2016).

Development of individual therapy occupational programs (IoT) and impacts on Neurocognition, Symptoms and Social Functioning of Patients with Schizophrenia, Shimada et al, is IOT in psychiatric facilities can improve psychosocial treatment of 
schizophrenia, improve cognition and symptoms in patients with schizophrenia (Shimada, Nishi, Yoshida, Tanaka, \& Kobayashi, 2016). The results of the Shimada, et al study were mixed effect models, indicating that the Occupational Group Therapy (GOT) + Individual Okupaei Therapy (IoT) shows a significant increase in verbal memory memory that works, verbal fluency, attention and composite score MMAS-8 score ( $\mathrm{p}<0.01)$ compared to GOT only. Patients on GOT + IoT showed a significant increase in the Japanese version of the Client Satisfaction Questionnaire-8 (CSQ-8J) (Shimada, Ohori, Inagaki, \& Shimooka, 2018). Independence of patients function Tanaka, et al shows that early occupational therapy can improve functional independence in acute schizophrenic patients (Tanaka, Yotsumoto, Tatsumi, Maeda, \& Hashimoto, 2014). Occupational Therapy of daily activities (ADL), Eklund, et al, describes satisfaction with daily activity activities and selfassessed health, and functional grouping effects reduce dependence on improvement findings in symptoms. Although the occupational therapy group ADL was lower the results than the Balance Everyday Life (BEL) group (Eklund, Tjörnstrand, Sandlund, \& Argentzell, 2017).

\section{DISCUSSION}

There are similarities in the results of research on the Occupational Occupational Therapy Program (IoT) and Occupational Group Therapy (GOT) better than GOT alone with significant results on improving cognitive function, including verbal memory, working memory, verbal ability. These findings provide preliminary support for the feasibility of applying IoT and effectiveness to improve cognition and symptoms in patients with schizophrenia with the same measurement tools, namely Short Cognition Assessment in Japanese-Schizophrenia (BACS-J), Positive and Negative Syndrome Scale (PANSS) . The research was carried out by the same author, but with different teams and at times there were additional assessments on the Japanese version of Intrinsic Motivation Inventory (IMI-J), Morisky-8 Drug Compliance Scale (MMAS-8), and the Japanese version of the Satisfaction Questionnaire Client-8 (CSQ-8J).

There is a similarity in the provision of interventions, namely occupational therapy: work activities but with a positive impact on varied schizophrenic patients, namely increasing independence, social work, social skills (social performance, interpersonal function), and decreasing psychiatric symptoms both negative and positive symptoms with measuring instruments that are Interpersonal Function Scale (IFS) and Personal and Social Performance Scale (PSP), Functional independence is measured using Functional Independence Measurement (FIM), Psychiatric symptoms are also measured by the Short Psychiatric Rating Scale, Social Work Function Scale (SOFS), and self-prepared checklist of social skills, Behavioral
Assessment of Dys-executive Syndrome (BADS) as the main outcome of executive function Therapy Occupational Rehabilitation Direct Functional Assessment (DAFS-BR) as well as the Independent Life Skills Survey (ILSS-BR) as a secondary outcome, The Syndrome Scale (PANSS) is used to monitor the severity of symptoms, Scale for the assessment of positive and negative symptoms (SANS, SAPS)

There is 1 study that is different from occupational therapy results: lower work training in schizophrenic patient satisfaction than daily activity Occupational Therapy (ADL), [6] describing the BEL Group increased more than the CAU group from baseline to 16 weeks in primary outcomes in terms of activity involvement ( $\mathrm{p}<0.001)$, activity level $(\mathrm{p}=$ $0.036)$ and activity balance ( $p<0.042)$, satisfaction with each day.

The aim of a systematic review of occupational therapy is to review the clinical evidence of the effect of therapeutic occupational intervention in schizophrenia. Based on the results of the analysis there are many effects resulting from various combinations of occupational therapy interventions. So that it can be continued in subsequent studies.

Based on a review of the differences in journals, it was found that occupational therapist interventions had a lot that stated that the benefits for schizophrenic patients such as improving cognitive, social, occupational and patient welfare functions contributed to the quality of life of patients and were effective for treatment in schizophrenic patients. Expectations at health care facilities namely Mental Hospital maximize and develop occupational therapy so as to improve the quality of life for schizophrenic patients and reduce the rate of recurrence. There are several potential limitations that are subscribed to by a systematic review of occupational therapy that is $\mathrm{A}$ few journals are in accordance with the criteria, namely with a village-level, quasi-experimental controlled RCT study with the limits of the last 5 years Many journals were not in English.

\section{CONCLUSION}

There are several occupational therapy journals explaining the benefits that are almost the same as having a positive effect on schizophrenic patients, and occupational therapy is more on the type of work training activities focusing on the recognition of existing abilities in patients by providing work skills training, and many are given in combination with other therapies such as social management, Psychosocial Interventions on Interpersonal Functioning and Personal and Social, Occupational Goal Intervention. There is one therapeutic occupational journal with a different type, namely focusing on Activity Daily Living (ADL). Occupational therapy interventions have some significant positive effects, especially in the treatment of schizophrenia.

\section{REFERENCES}

Amico, M. L. D., Jaffe, L. E., \& Gardner, J. A. (2018). 
Evidence for Interventions to Improve and Maintain Occupational Performance and Participation for People With Serious Mental Illness : A Systematic Review.

Badan Penelitian dan Pengembangan Kesehatan. (2013). Riset Kesehatan Dasar (RISKESDAS) 2013. Laporan Nasional 2013, 1-384. https://doi.org/1 Desember 2013

Çakmak, S., Süt, H., Öztürk, S., Tamam, L., \& Bal, U. (2016). The Effects of Occupational Therapy and Psychosocial Interventions on Interpersonal Functioning and Personal and Social Performance Levels of Corresponding Patients. (9), 234-240. https://doi.org/10.5152/npa.2015.10130

Eklund, M., Tjörnstrand, C., Sandlund, M., \& Argentzell, E. (2017). Effectiveness of Balancing Everyday Life (BEL) versus standard occupational therapy for activity engagement and functioning among people with mental illness - a cluster RCT study. BMC Psychiatry, 17(1), 1-12. https://doi.org/10.1186/s12888017-1524-7

Foruzandeh, N., \& Parvin, N. (2012). Occupational therapy for inpatients with chronic schizophrenia: A pilot randomized controlled trial. https://doi.org/10.1111/j.17427924.2012.00211.x

Morris, K., Syed, A., Reid, G., \& Spencer, S. (2016). Occupational therapy delivered by specialists versus non- specialists for people with schizophrenia ( Protocol ). (10). https://doi.org/10.1002/14651858.CD012398 .www.cochranelibrary.com

Shimada, T., Nishi, A., Yoshida, T., Tanaka, S., \& Kobayashi, M. (2016). Development of an Individualized Occupational Therapy Programme and its Effects on the Neurocognition
, Symptoms and Social Functioning of Patients with Schizophrenia. 425-435. https://doi.org/10.1002/oti.1445

Shimada, T., Ohori, M., Inagaki, Y., \& Shimooka, Y. (2018). A multicenter, randomized controlled trial of individualized occupational therapy for patients with schizophrenia in Japan. 1-18.

Singh, U., \& Singh, B. (2018). Assessment and Management of Socio-Occupational Functioning of Persons with Chronic Schizophrenia : Effect of. 13(1), 189-198.

Singh, U., Singh, B., \& . S. (2019). Assessment and Management of Socio-Occupational Functioning of Persons with Chronic Schizophrenia: Effect of Social Skills Training. Journal of Psychosocial Research, 13(1), 189-198. https://doi.org/10.32381/jpr.2018.13.01.18

Tanaka, C., Yotsumoto, K., Tatsumi, E., Maeda, K., \& Hashimoto, T. (2014). Improvement of functional independence of patients with acute schizophrenia through early occupational therapy: a pilot quasi- experimental controlled study. 7-10. https://doi.org/10.1177/0269215514521440

Videbeck, Sheila, L. (2008). Buku Ajar Keperawatan Jiwa. Jakarta: Penerbit Buku Kedokteran EGC.

Vizzotto, A. D. B., Celestino, D. L., Buchain, P. C., Oliveira, A. M., Oliveira, G. M. R., Di Sarno, E. S., ... Elkis, H. (2016). A pilot randomized controlled trial of the Occupational Goal Intervention method for the improvement of executive functioning in patients with treatment-resistant schizophrenia. Psychiatry Research, 245, 148156.

https://doi.org/10.1016/j.psychres.2016.05.0 11 